Article

\title{
Flexible Carbon Nanotube Modified Separator for High-Performance Lithium-Sulfur Batteries
}

\author{
Bin Liu ${ }^{1}$, Xiaomeng $\mathrm{Wu}^{1}{ }^{1}$, Shan Wang ${ }^{1, *}$, Zhen Tang ${ }^{1}$, Quanling Yang ${ }^{1}$, Guo-Hua $\mathrm{Hu}^{1,2}$ \\ and Chuanxi Xiong ${ }^{1, *}$ \\ 1 State Key Laboratory of Advanced Technology for Materials Synthesis and Processing, \\ School of Materials Science and Engineering, Wuhan University of Technology, Wuhan 430070, China; \\ kingingben@outlook.com (B.L.); wxm07156@163.com(X.W.); zhentang@whut.edu.cn (Z.T.); \\ yangql@whut.edu.cn (Q.Y.); hufrance@hotmail.com (G.-H.H.) \\ 2 Laboratory of Reactions and Process Engineering (LRGP, CNRS UMR 7274), CNRS-University of Lorraine, \\ 1rue Grandville, BP 20451, 54001 Nancy, France \\ * Correspondence: shanwang@whut.edu.cn (S.W.); cxiong@whut.edu.cn (C.X.); Tel.: +86-027-8738-7481 (C.X.)
}

Received: 10 July 2017; Accepted: 21 July 2017; Published: 26 July 2017

\begin{abstract}
Lithium-sulfur (Li-S) batteries have become promising candidates for electrical energy storage systems due to their high theoretical specific energy density, low cost and environmental friendliness. However, there are some technical obstacles of lithium-sulfur batteries to be addressed, such as the shuttle effect of polysulfides. Here, we introduced organically modified carbon nanotubes (CNTs) as a coating layer for the separator to optimize structure and enhance the performance of the Li-S battery. The results showed that the cell with a CNTs-coated separator exhibited an excellent cycling performance. Compared to the blank separator, the initial discharge capacity and the capacity after 100 cycles for the CNTs-coated separator was increased by $115 \%$ and 161\%, respectively. Besides, according to the rate capability test cycling from $0.1 \mathrm{C}$ to $2 \mathrm{C}$, the battery with a CNTs-coated separator still released a capacity amounting to $90.2 \%$ of the initial capacity, when the current density returned back to $0.1 \mathrm{C}$. It is believed that the organically modified CNTs coating effectively suppresses the shuttle effect during the cycling. The employment of a CNTs-coated separator provides a promising approach for high-performance lithium-sulfur batteries.
\end{abstract}

Keywords: Lithium-sulfur batteries; carbon nanotubes; separators; shuttle effect

\section{Introduction}

Nowadays, rechargeable lithium batteries have been widely used in portable electronics, electric vehicles and various energy storage devices. Lithium-sulfur (Li-S) batteries have been considered to be one of the most promising choices for next generation, high-energy rechargeable batteries due to their high theoretical energy density. For lithium-ion batteries with graphite as negative electrodes, the theoretical energy densities are typically limited to around $400 \mathrm{Wh} / \mathrm{kg}$ or $1400 \mathrm{Wh} / \mathrm{L}$ [1,2], while the theoretical energy densities of lithium-sulfur batteries are calculated to be $2500 \mathrm{Wh} / \mathrm{kg}$ or $2800 \mathrm{Wh} / \mathrm{L}[3,4]$. Also sulfur offers a theoretical capacity of $1675 \mathrm{mAh} / \mathrm{g}$, which is higher than those of transition-metal oxide electrode materials by an order of magnitude [5]. Additionally, the merits of abundant reserves, low cost and environmental friendliness of sulfur are greatly in favor of large-scale commercialization of Li-S batteries.

In spite of these advantages, there are still great challenges limiting the practical application of Li-S batteries. On one hand, there is low utilization of the active materials in the cathode since both sulfur and its discharge product $\mathrm{Li}_{2} \mathrm{~S}$ are electronically and ionically insulating. On the other hand, due to the soluble characteristics and thus the shuttle behavior of the polysulfide intermediates $\left(\mathrm{Li}_{2} \mathrm{~S}_{x}, 4 \leq x \leq 8\right)$, which are generated during the charge/discharge process, irreversible capacity loss 
is commonly unavoidable. This consequently leads to low coulombic efficiency and poor cycle life for Li-S batteries [6].

To address these key issues, great efforts have been devoted in the past decades mainly toward improving the electrochemical performance of the sulfur cathode. A variety of carbon materials such as carbon nanofibers [7], graphene [8,9] and porous carbon [10] have been employed to fabricate sulfur/carbon composite cathode to enhance the conductivity of sulfur as well as to suppress the diffusion of dissolved polysulfides out of the cathode. Also conductive polymers have been adopted and have demonstrated their role in improving the electrochemical performance of the sulfur cathode [11]. However, the enhanced electrochemical performance is usually compromised by the lower sulfur loading mass in the cathode, which greatly reduces the practical energy density of Li-S batteries. Alternatively, a promising strategy based on modifying the separator with conductive materials (e.g., Super P, Ketjen Black) has been recently proposed and demonstrated its great effectiveness on improving the rate capability and cycle life of Li-S batteries [12,13]. As an essential part of batteries, separators are placed between anode and cathode to prevent physical contact of the electrodes during the free ion transfer process. The properties of separators such as composition, porosity and wettability can affect the energy density, cycle life and safety of the battery [14]. It was claimed that the conductive coating or layer on separator could facilitate electron or ion transport, enhance electrolyte uptake, and inhibit the shuttle effect of polysulfides [15].

Owing to their outstanding electrical conductivity, one-dimensional carbon nanotubes (CNTs) have attracted considerable interest in the field of the lithium sulfur battery, especially in terms of the electrodes $[16,17]$. Few studies have involved coating the separator with CNTs due to their entanglement, poor solubility and dispersion in solvents. Chung et al. fabricated CNTs-coated separator by a simple vacuum filtration technique, and the results showed that Li-S battery with the modified separator possessed high discharge capacity, excellent rate performance and long cycle life [18]. However, low efficiency and safety problems (i.e., separator rupture) may be involved in the vacuum filtration process. Therefore, it is highly desirable to design a facile but efficient route to apply the CNTs coating on the commercial separator.

In this work, flexible carbon nanotubes were first prepared by oxidation followed by a grafting route. Then the modified CNTs were spin coated onto the surface of the commercial separator, and assembled into Li-S battery. The electrochemical performance of lithium-sulfur batteries was comprehensively studied. The hydrophilic groups and reticular structure in the organically modified CNTs coating layer effectively suppressed the shuttle effect by trapping dissolved long-chain lithium polysulfides $\left(\mathrm{Li}_{2} \mathrm{~S}_{x}, x=4,6,8\right)$. The introduction of organically modified CNTs enhanced the thermal shrinkage property of the separator, which is beneficial for its practical application. Besides, the conductive nature of CNTs also benefited the decrease of the internal resistance. To sum up, modified CNTs-coated separators are promising for high performance lithium-sulfur batteries.

\section{Results and Discussion}

\subsection{Morphology and Structural Characterization}

As shown in Figure 1a, the organic modification process of carbon nanotubes consisted of three successive steps. Carbon nanotubes were first oxidized by a mixed, concentrated acid of $\mathrm{H}_{2} \mathrm{SO}_{4}$ and $\mathrm{HNO}_{3}$, resulting in a great amount of hydrophilic groups including carboxyl and hydroxyl on the surface of carbon nanotubes. An organic silane $\left(\left(\mathrm{CH}_{3} \mathrm{O}\right)_{3} \mathrm{Si}_{(}\left(\mathrm{CH}_{2}\right)_{3} \mathrm{~N}^{+}\left(\mathrm{CH}_{3}\right)_{2}\left(\mathrm{C}_{18} \mathrm{H}_{37}\right) \mathrm{Cl}^{-}\right)(\mathrm{DC} 5700)$ was adopted to couple and a long-chain protonic acid containing a polyethylene glycol segment $\left(\mathrm{C}_{9} \mathrm{H}_{19}-\mathrm{C}_{6} \mathrm{H}_{4}-\mathrm{O}\left(\mathrm{CH}_{2} \mathrm{CH}_{2} \mathrm{O}\right)_{10} \mathrm{SO}_{3}{ }^{-} \mathrm{Na}^{+}\right)$(NPES) was applied to exchange ion successively $[19,20]$. The as-obtained products were spin coated onto the commercial Celgard 2325 separator and the coated separators were assembled into lithium-sulfur batteries. As shown in Figure 1b, the coated separators were designed to trap and prevent lithium polysulfides from crossing the separators, which would effectively suppress the shuttle effect. 
(a)

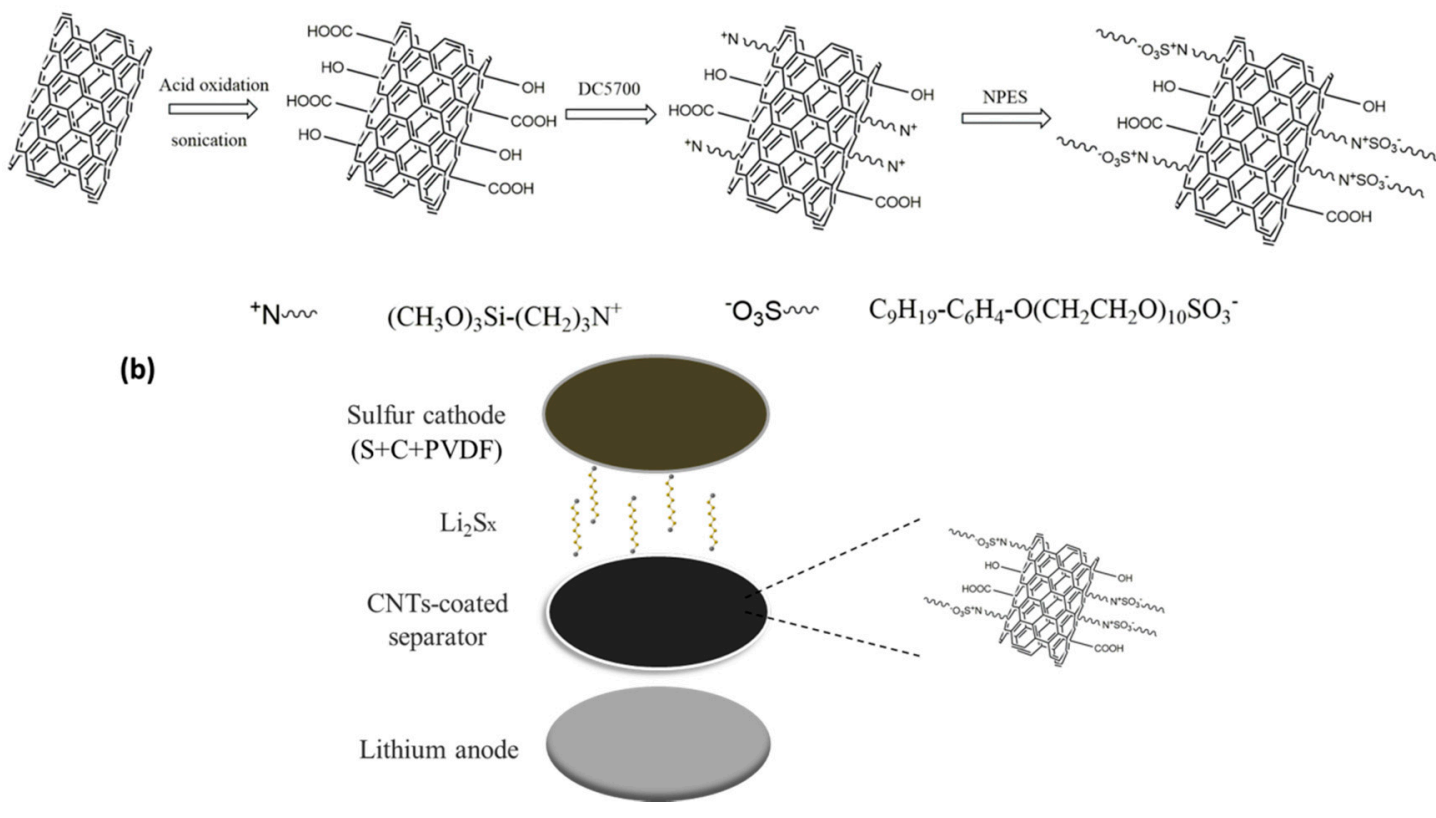

Figure 1. Schematic illustration of (a) organic modification process of carbon nanotubes and (b) cell configuration of lithium-sulfur (Li-S) batteries.

Typical transmission electron microscope (TEM) images of the original and oxidized CNTs are shown in Figure 2a,b. Comparing to the original CNTs, it is found that less entanglements are present for the oxidized CNTs. This could be due to the successful introduction of some hydrophilic groups such as hydroxyl and carboxyl in the oxidized process, consequently leading to an improved dispersion of CNTs in water. Figure 2c shows the TEM of the synthesized CNTs. It can be seen that the CNTs are surrounded by a shell structure with a thickness of about $1 \mathrm{~nm}$, which is the organic shell formed by DC 5700 and NPES long-chain molecules grafted on the CNTs surface. After graft modification, the entanglement of CNTs reduced greatly. The CNTs could be easily dispersed in some solvents such as chloroform, and form a homogeneous slurry state, which facilitates the coating technique.
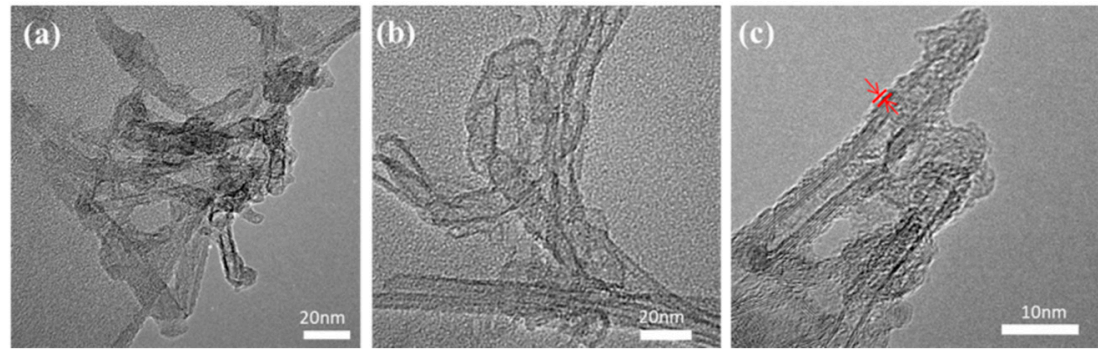

Figure 2. TEM images of (a) original carbon nanotubes (CNTs), (b) oxidized CNTs and (c) organically modified CNTs.

Figure 3 compares the Fourier transform infrared (FTIR) spectra of the oxidized and organically modified CNTs. Some additional characteristic peaks can be distinctively observed in the spectrum of the organically modified CNTs. Specifically, characteristic peaks around $2925 \mathrm{~cm}^{-1}$ and $2856 \mathrm{~cm}^{-1}$ corresponded to the stretching vibration of methyl and methylene, while the peak at $1467 \mathrm{~cm}^{-1}$ represented the bending vibration of methylene. The peak at $1251 \mathrm{~cm}^{-1}$ was characteristic of the $-\mathrm{SO}_{3}$ group and the peaks of $1170 \mathrm{~cm}^{-1}$ and $1029 \mathrm{~cm}^{-1}$ were attributed to the Si-O-Si vibration. Besides, it was found that the vibration strength of the hydroxyl peak at $3450 \mathrm{~cm}^{-1}$ reduced for the organically 
modified CNTs, indicating that the amount of hydroxyl groups decreased due to the reaction between the CNTs and the organic silane DC 5700. Combined with the chemical structure of the DC 5700 and NPES, it was reasonably deduced that the organic modifiers have been successfully grafted onto the walls of CNTs in this study.

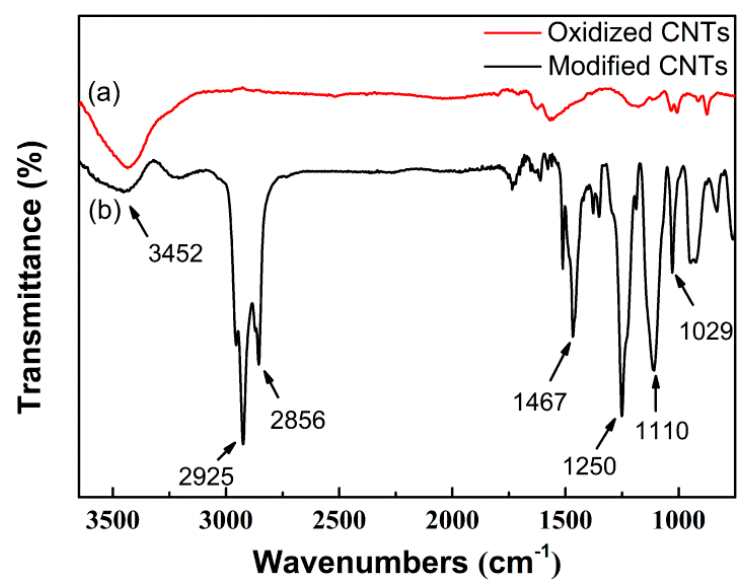

Figure 3. FTIR spectra of (a) oxidized CNTs and (b) organically modified CNTs.

Figure $4 \mathrm{a}$,b show the scanning electron microscope (SEM) images of the blank and CNTs-coated separators, respectively. Typically, the blank separator displayed uniform slit-like micropores with a length of about $280 \mathrm{~nm}$ and a width of about $70 \mathrm{~nm}$. It is known that these nanosized micropores could provide a convenient channel for transporting the free lithium ion. Unfortunately, it was also said that this type of micropore allowed the shuttle of the dissolved polysulfides during the charge/discharge process, which consequently deteriorated the rate performance and shortened the cycle life [21]. While for the CNTs-coated separator, it is clear that the tubular carbon materials were uniformly adhered to the surface of the separator and the original porous structure was entirely covered. The long CNT tubes intertwined with each other and constructed a large number of nanosized pore structure in the coating layer. According to previous reports, this structure could facilitate the storage of the polysulfide intermediates [18].
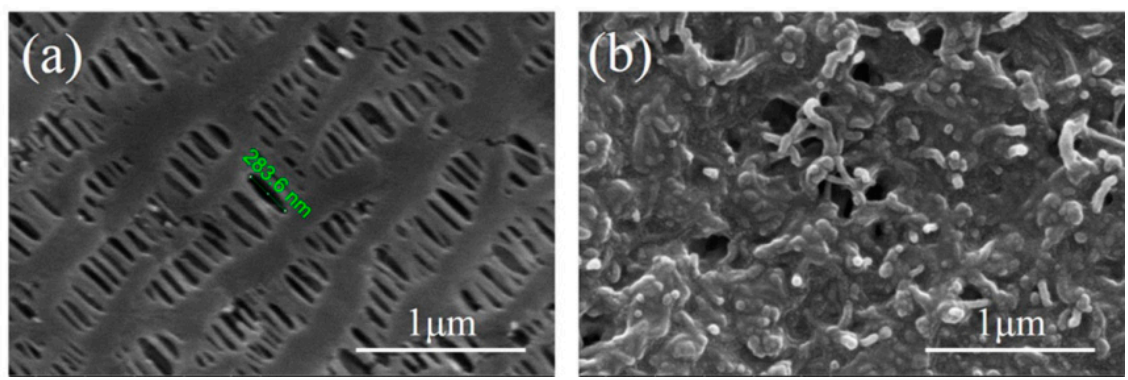

Figure 4. SEM images of (a) blank separator and (b) CNTs-coated separator.

Essentially, the surface chemistry property of separator plays an important role in controlling the diffusion of polysulfides. Considering the water contact angle (WCA) can reflect the polarity of the separators, WCA measurements were carried out as shown in Figure 5. The WCA of the blank separator was approximately $114.0^{\circ}$ (Figure 5a), which associated well with the hydrophobic nature of the polypropylene-based separator. In contrast, the water droplet quickly spread as soon as it contacted with the surface of the CNTs-coated separator, and the WCA measured at $10 \mathrm{~s}$ was only about $15^{\circ}$, suggesting a good hydrophilicity. The result was in consistence with the previous FTIR 
analysis, indicating the presence of long-chain organic modifier and abundant hydrophilic groups including hydroxyl and carboxyl groups on the walls of the CNTs. And it is known that hydrophilicity of separator would benefit for the suppression of shuttle effect by absorbing polysulfides [8,22].

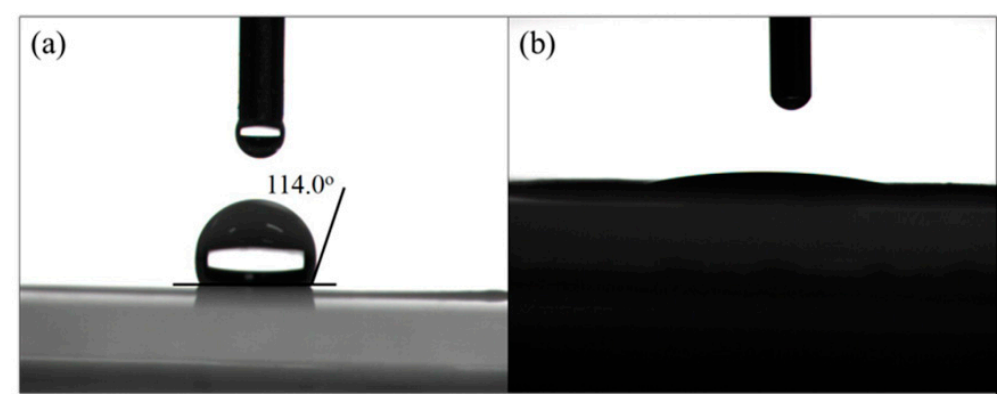

Figure 5. Water contact angles of (a) blank separator and (b) CNTs-coated separator.

Considering the main function of separators in preventing physical contact of anode and cathode, separators should be stable at high temperature, which is common in practical application. In order to assess the thermal stability of the separators at high temperatures, the separators were treated at different temperatures from $120^{\circ} \mathrm{C}$ to $155^{\circ} \mathrm{C}$ for $30 \mathrm{~min}$ to calculate the shrinkage percentages as shown in Table 1. It is clear that the thermal shrinkage of the CNTs-coated separator was less than that of blank separator under the same thermal circumstance, suggesting an improved stability upon the CNTs modification. Take $150{ }^{\circ} \mathrm{C}$ as an example, the shrinkage of the CNTs-coated separator is $25 \%$, which is much lower than that of blank separator (38.3\%). It was suggested that the CNTs skeleton could prevent the closure of micropores and thus ensure the stability of separators at high temperature.

Table 1. Thermal shrinkage of blank separator and CNTs-coated separator.

\begin{tabular}{cccccc}
\hline \multirow{2}{*}{ Separator } & \multicolumn{5}{c}{ Shrinkage of Separator (\%) } \\
\cline { 2 - 6 } & $\mathbf{1 2 0}{ }^{\circ} \mathbf{C}$ & $\mathbf{1 3 0}{ }^{\circ} \mathbf{C}$ & $\mathbf{1 4 0}{ }^{\circ} \mathbf{C}$ & $\mathbf{1 5 0}^{\circ} \mathbf{C}$ & $\mathbf{1 5 5}^{\circ} \mathbf{C}$ \\
\hline Blank & 6.7 & 10.3 & 21.7 & 38.3 & 43.0 \\
CNTs-coated & 5.0 & 9.3 & 16.0 & 25.0 & 35.0 \\
\hline
\end{tabular}

\subsection{Electrochemical Performance}

The cyclic voltammetry was applied to clarify the mechanism of lithium-sulfur batteries as shown in Figure 6. The cyclic voltammogram of the CNTs-coated separator owned three distinct peaks including two reduction peaks and one oxidation peak. Compared to the cell with commercial separator (not shown here), no additional peaks were observed for the cell loaded with the CNTs-coated separator, indicating that the CNTs coating layer did not participate in the redox reaction during the charge/discharge process. Generally, the reduction peaks around $2.3 \mathrm{~V}$ and $2.0 \mathrm{~V}$ corresponded to the conversion from $S_{8}$ to long-chain polysulfides $\left(\operatorname{Li}_{2} S_{x}, 4 \leq x \leq 8\right)$ and the formation of $\mathrm{Li}_{2} \mathrm{~S}_{2} / \mathrm{Li}_{2} \mathrm{~S}$, respectively, while the oxidation peak centered around $2.5 \mathrm{~V}$ suggested the typical elemental sulfur converting process [23-25]. Besides, it should be noted that the cyclic voltammograms in Figure 6 almost coincided with each other in the first three cycles, indicating a high reversibility of electrochemical reaction for the battery with the CNTs-coated separator, which could be ascribed to the suppression of shuttle effect and stability of the battery. 


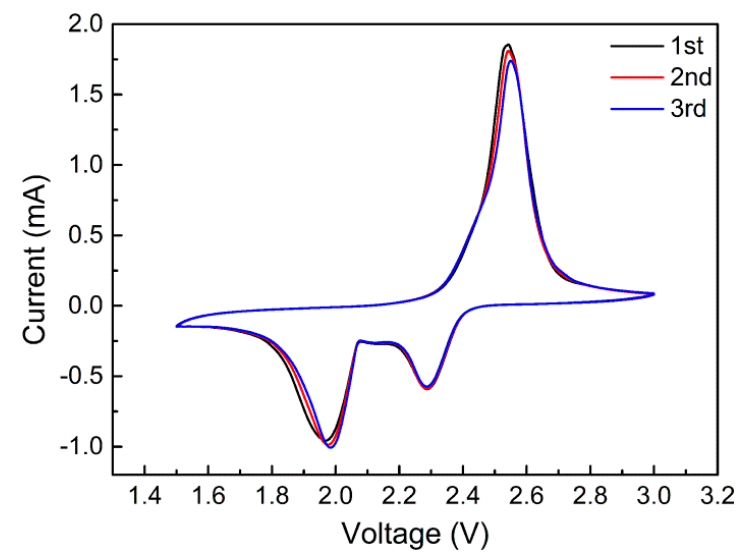

Figure 6. Cyclic voltammograms of the cells with CNTs-coated separator.

Figure 7a compares the discharge capacity of the Li-S batteries with the blank separator and CNTs-coated separator. It was found that the cell with the CNTs-coated separator delivered an initial discharge capacity as high as $1179 \mathrm{mAh} / \mathrm{g}$ at $0.1 \mathrm{C}$ while the cell with the blank separator only delivered an initial value of $547 \mathrm{mAh} / \mathrm{g}$. In addition, the discharge capacity of the CNTs-coated and blank separators remained at $595 \mathrm{mAh} / \mathrm{g}$ and $228 \mathrm{mAh} / \mathrm{g}$ after 100 cycles, suggesting the capacity retention rates of $50.6 \%$ and $41.7 \%$, respectively. This suggested that modification of the separator with the CNTs coating improved not only the initial discharge capacity but also the capacity retention rate. The improvement should be ascribed to the CNTs coating, which was capable of trapping long-chain polysulfides during the cycling.
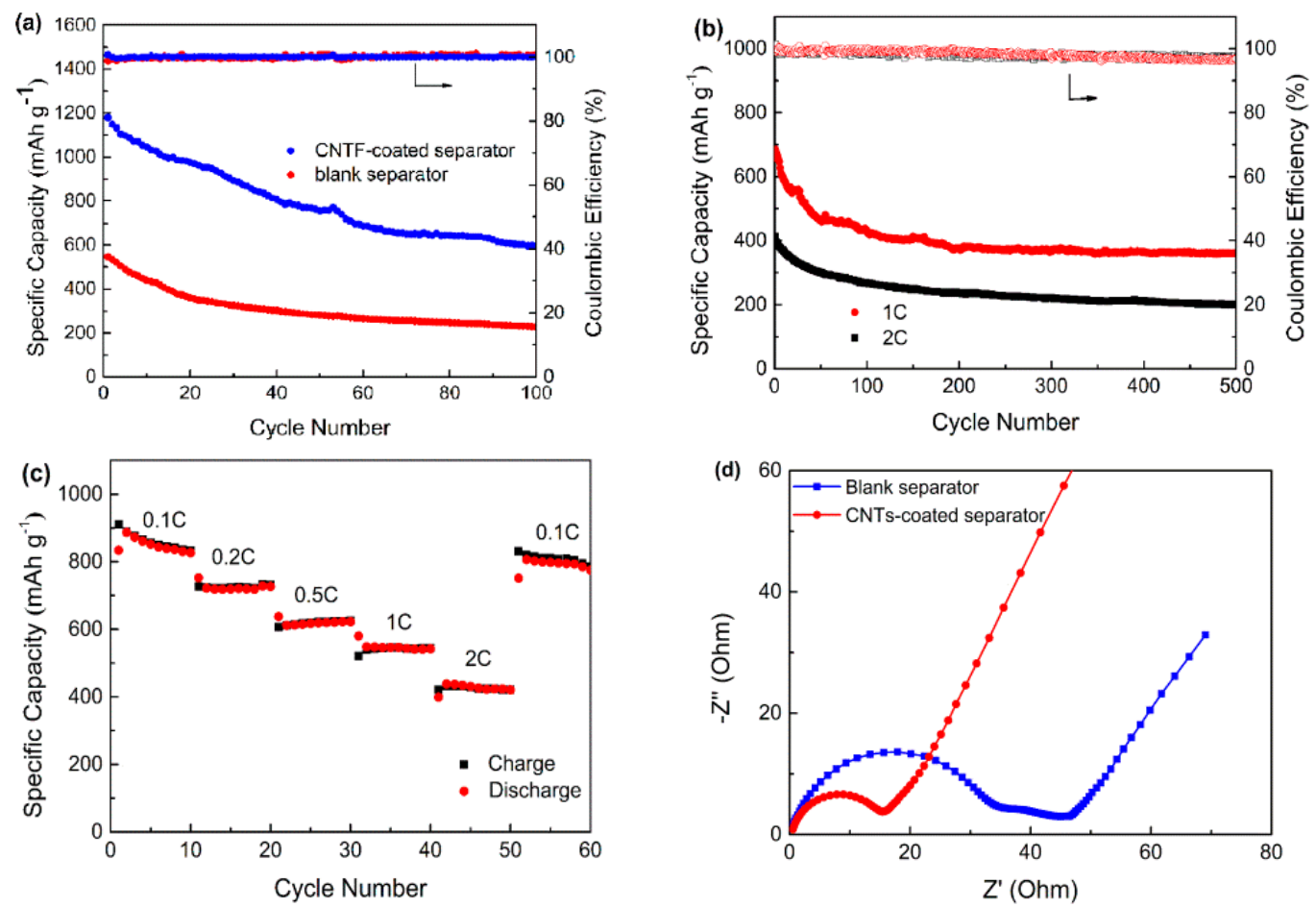

Figure 7. (a) Cycling performance of the blank separator and CNTs-coated separator at 0.1C; (b) Cycling performance of the CNTs-coated separator at the rates of $1 \mathrm{C}$ and 2C; (c) Rate performance of the Li-S batteries with the CNTs-coated separator; (d) EIS data of the cells with the blank separator and CNTs-coated separator. 
The cycling performance of the Li-S battery with the CNTs-coated separator at high rates was further analyzed as shown in Figure $7 \mathrm{~b}$. The reversible capacity of the battery dropped from $684 \mathrm{mAh} / \mathrm{g}$ to $361 \mathrm{mAh} / \mathrm{g}$ at $1 \mathrm{C}$ in 500 cycles, with a coulombic efficiency of $96.7 \%$ on average and a capacity retention rate of $52.8 \%$. When the rate increased to $2 \mathrm{C}$, the initial discharge capacity of the battery was $414 \mathrm{mAh} / \mathrm{g}$ and remained $200 \mathrm{mAh} / \mathrm{g}$ after 500 cycles, suggesting an average capacity decay rate of only $0.1 \%$ per cycle. The coulombic efficiency also kept as high as $96.8 \%$. The result is encouraging since it suggested the CNTs coating in the work could maintain the structural stability of the battery. That is to say, it would not collapse, even as the charge and discharge occurs rapidly, consequently leading to a long cycle life of the Li-S battery at high current density. Figure 7c presents the rate performance of the Li-S batteries with the CNTs-coated separator. With an initial value of $887 \mathrm{mAh} / \mathrm{g}$ at $0.1 \mathrm{C}$, the capacity remained around $720,620,545$ and $430 \mathrm{mAh} / \mathrm{g}$ at $0.2 \mathrm{C}, 0.5 \mathrm{C}, 1 \mathrm{C}$ and $2 \mathrm{C}$ in 10 cycles, respectively, and returned back to $800 \mathrm{mAh} / \mathrm{g}$ when the rate was $0.1 \mathrm{C}$. The excellent reversibility of the capacity as high as $90.2 \%$ supported strongly again that the Li-S battery with CNTs-coated separator was highly stable and reversible.

Electrochemical impedance spectra (EIS) of the battery with and without CNTs-coated separator are shown in Figure 7d. The Nyquist plots for this battery consisted of a semicircle in high frequency region, another in medium-to-low frequency region and an oblique line in the low-frequency region, which represented the resistance from a passivation film $\left(R_{t}\right)$, the charge-transfer resistance $\left(R_{c t}\right)$ and Warburg impedance, respectively [26,27]. According to the previous reports, the diameter size of the semicircle and slope size of the oblique line represented the value of impedance. It is clear that $R_{c t}$ and Warburg impedance are similar to each other for the different battery, while the $R_{t}$ from a passivation film of the battery with CNTs-coated separator is less than that of the battery with blank separator. The results indicated that CNTs coating could significantly reduce the $R_{t}$, due to the conductive nature of CNTs and the enhanced electrolyte absorption capability. Similar to the previous study by other researchers, the enhanced electrical conductivity of the battery with CNTs coated separator led to a better rate capability and a high reversible capacity than that with blank separator [28]. The result was consistent with the preceding enhanced cycling performance.

\section{Materials and Methods}

\subsection{Materials}

Multi-walled carbon nanotubes (CNTs) were provided by Chengdu Organic Chemicals Co., Ltd. (Chengdu, Sichuan, China) Ethanol, chloroform, concentrated sulfuric acid $\left(\mathrm{H}_{2} \mathrm{SO}_{4}, 98 \%\right)$, and concentrated nitric acid $\left(\mathrm{HNO}_{3}, 65 \%\right)$ were purchased from Sinopharm Chemical Reagent Co., Ltd. (Shanghai, China). Polysiloxane quaternary ammonium salt DC5700 $\left[\left(\mathrm{CH}_{3} \mathrm{O}\right)_{3} \mathrm{Si}\left(\mathrm{CH}_{2}\right)_{3} \mathrm{~N}^{+}\left(\mathrm{CH}_{3}\right)_{2}\left(\mathrm{C}_{18} \mathrm{H}_{37}\right) \mathrm{Cl}^{-}\right]$in methanol (40\%) was provided by Gelest (Morrisville, PA, USA). Sulfonate salt NPES $\left[\mathrm{C}_{9} \mathrm{H}_{19} \mathrm{C}_{6} \mathrm{H}_{4} \mathrm{O}\left(\mathrm{CH}_{2} \mathrm{CH}_{2} \mathrm{O}\right){ }_{10} \mathrm{SO}_{3}{ }^{-} \mathrm{K}^{+}\right]$was obtained from Aldrich (Shanghai, China). Sublimed sulfur was purchased from Sinopharm Chemical Reagent Co., Ltd. (Shanghai, China). Celgard 2325 from Celgard, LLC (Charlotte, NC, USA) was adopted as separator. Bis(trifluoromethane) sulfonamide lithium(LiTFSI), lithium nitrate $\left(\mathrm{LiNO}_{3}, 99.99 \%\right.$ trace metals basis), $n$-butyl alcohol (99\%), N-methyl-2-pyrrolidone (NMP, 99\%), 1,3-dioxolane (DOL) and 1,2-dimethoxyethane (DME) were obtained from Aladdin (Shanghai, China).

\subsection{Preparation of CNTs-Coated Separator}

Typically, CNTs were first oxidized in a mixture of concentrated $\mathrm{H}_{2} \mathrm{SO}_{4}$ and $\mathrm{HNO}_{3}$ with a volume ratio of $3: 1$ at $50{ }^{\circ} \mathrm{C}$ for $3 \mathrm{~h}$. The suspension was then diluted with deionized water, followed by a centrifugation to remove the supernatant acid. The remaining solids were washed repeated with deionized water until the $\mathrm{pH}$ value reached about 3. DC5700 was then added into the aqueous solution of oxidized CNTs to render them cationic. The mixture was sonicated for $2 \mathrm{~h}$, washed with water and anhydrous ethanol successively, and dried at $70{ }^{\circ} \mathrm{C}$ for $6 \mathrm{~h}$. Subsequently, the resultants and a certain 
proportion of NPES were mixed in the chloroform solution. The mixture was stirred for $5 \mathrm{~h}$ at room temperature to ensure a full reaction, followed by a dialysis for $24 \mathrm{~h}$ in the deionized water bath to completely remove the $\mathrm{KCl}$ salt and excess sulfonate salt. Finally, the product was vacuum-dried at $70{ }^{\circ} \mathrm{C}$ for $24 \mathrm{~h}$ to obtain the modified CNTs.

An appropriate amount of CNTs was dissolved in the chloroform to prepare the CNTs slurry. Then a small amount of CNTs slurry was spin-coated onto the surface of the virgin separator. Before the coating operation, the virgin separator was cleaned with anhydrous ethanol in an ultrasonic bath to remove any contamination. The coated separator was then dried in a vacuum oven at $50{ }^{\circ} \mathrm{C}$ for $24 \mathrm{~h}$.

\subsection{Characterizations}

TEM images were made on a JEM 2100F field emission transmission electron microscope (Japan Electronics Co., Ltd., Tokyo, Japan). FTIR analysis was carried out using a Nicolet 6700 Fourier transform infrared spectrometer (Thermo Fisher Scientific Inc., Waltham, WA, USA). The surface morphologies of the virgin and coated separators were observed using a field emission scanning electron microscope (SEM, QUANTA FEG 450, FEI, Hillsboro, OR, USA). The water contact angles of the separators were measured by a JC 2000C contact angle measuring instrument (Powereach, Shanghai, China). The thermal shrinkage ratio $S$ was calculated according to the expression $S=\left(L_{0}-L\right) / L_{0} \times 100 \%$, where $L_{0}$ and $L$ are the lengths of the separators before and after the thermal treatment, respectively.

The CR2032 coin cells were assembled in an argon-filled glove box to evaluate the electrochemical performance of the virgin and CNTs-coated separator. The electrolyte was made up of $1 \mathrm{M}$ bis(triuoromethanesulfonyl)imide (LiTFSI) in dioxolane/dimethoxyethane solution (DOL/DME, $V / V=1: 1$ ) with 1 wt $\% \mathrm{LiNO}_{3}$ additive. The cathode was prepared by mixing elemental sulfur, acetylene black and poly(vinylidene fluoride) with a weight ratio of 3:2:1 in $N$-methyl-2-pyrrolidinone. The slurry was coated on the aluminum foil and dried under vacuum to form the working cathode. Lithium metal foil was adopted as the anode. Cycling performance of the batteries was analyzed using a cell testing system (LAND CT2001A, Wuhan LAND electronics, Wuhan, China). Both the cyclic voltammetry curves (CV) and AC impedance of the battery were measured using a CHI 660E electrochemical workstation ( $\mathrm{CH}$ Instruments, Shanghai, China). The voltage range was controlled in $1.5 \sim 3 \mathrm{~V}$ and the scanning rate was $0.1 \mathrm{mV} / \mathrm{s}$. For the $\mathrm{AC}$ impedance measurement, the scanning frequency range was controlled from 0.01 to $100 \mathrm{kHz}$, with an AC potential amplitude of $5 \mathrm{mV}$ at the open-circuit potential.

\section{Conclusions}

In summary, we proposed a novel and effective method for the modification of separators in lithium-sulfur batteries. Grafted long-chain molecules and tube-like CNTs enable the formation of micropores in CNTs, which could accommodate polysulfides. The hydrophilic groups help trapping polysulfides to suppress shuttle effect. The organically modified CNTs coating also facilitate the battery with a high temperature stability. In addition, the conductive nature of CNTs also contributes to a decrease in internal resistance. The performance of the battery is improved significantly after adopting our method, indicating new avenues for practical lithium-sulfur batteries.

Acknowledgments: Financial support from the National Science Foundation of China (grant No. 51673154) is greatly acknowledged.

Author Contributions: Bin Liu conceived and designed the experiments; Bin Liu, Xiaomeng Wu and Zhen Tang performed the experiments and analyzed the data; Bin Liu wrote the paper. Quanling Yang and Guo-Hua HU edited the paper. Shan Wang and Chuanxi Xiong proposed the project and supervised the paper.

Conflicts of Interest: The authors declare no conflicts of interest. 


\section{References}

1. Adelhelm, P.; Hartmann, P.; Bender, C.L.; Busche, M.; Eufinger, C.; Janek, J. From lithium to sodium: Cell chemistry of room temperature sodium-air and sodium-sulfur batteries. Beilstein J. Nanotechnol. 2015, 6, 1016-1055. [CrossRef] [PubMed]

2. Whittingham, M.S. Ultimate limits to intercalation reactions for lithium batteries. Chem. Rev. 2014, 114, 11414-11443. [CrossRef] [PubMed]

3. Evers, S.; Nazar, F.L. New Approaches for High Energy Density Lithium-Sulfur Battery Cathodes. Acc. Chem. Res. 2013, 46, 1135-1143. [CrossRef] [PubMed]

4. Seh, Z.W.; Sun, Y.; Zhang, Q.; Cui, Y. Designing high-energy lithium-sulfur batteries. Chem. Soc. Rev. 2016, 45, 5605-5634. [CrossRef] [PubMed]

5. Manthiram, A.; Fu, Y.; Chung, S.H.; Zu, C.; Su, Y.S. Rechargeable lithium-sulfur batteries. Chem. Rev. 2014, 114, 11751-11787. [CrossRef] [PubMed]

6. Chen, L.; Shaw, L.L. Recent advances in lithium-sulfur batteries. J. Power Sources 2014, 267, 770-783. [CrossRef]

7. Lee, J.S.; Kim, W.; Jang, J.; Manthiram, A. Sulfur-Embedded Activated Multichannel Carbon Nanofiber Composites for Long-Life, High-Rate Lithium-Sulfur Batteries. Adv. Energy Mater. 2016, 7, 1601943. [CrossRef]

8. Wang, X.; Wang, Z.; Chen, L. Reduced graphene oxide film as a shuttle-inhibiting interlayer in a lithium-sulfur battery. J. Power Sources 2013, 242, 65-69. [CrossRef]

9. Jin, K.; Zhou, X.; Liu, Z. Graphene/Sulfur/Carbon Nanocomposite for High Performance Lithium-Sulfur Batteries. Nanomaterials 2015, 5, 1481-1492. [CrossRef] [PubMed]

10. Chen, M.; Jiang, S.; Cai, S.; Wang, X.; Xiang, K.; Ma, Z.; Song, P.; Fisher, A.C. Hierarchical porous carbon modified with ionic surfactants as efficient sulfur hosts for the high-performance lithium-sulfur batteries. Chem. Eng. J. 2017, 313, 404-414. [CrossRef]

11. Gao, H.; Lu, Q.; Yao, Y.; Wang, X.; Wang, F. Significantly Raising the Cell Performance of Lithium Sulfur Battery via the Multifunctional Polyaniline Binder. Electrochim. Acta 2017, 232, 414-421. [CrossRef]

12. Yao, H.; Yan, K.; Li, W.; Zheng, G.; Kong, D.; Seh, Z.W.; Narasimhan, V.K.; Liang, Z.; Cui, Y. Improved lithium-sulfur batteries with a conductive coating on the separator to prevent the accumulation of inactive S-related species at the cathode-separator interface. Energy Environ. Sci. 2014, 7, 3381-3390. [CrossRef]

13. Chung, S.-H.; Manthiram, A. Bifunctional Separator with a Light-Weight Carbon-Coating for Dynamically and Statically Stable Lithium-Sulfur Batteries. Adv. Funct. Mater. 2014, 24, 5299-5306. [CrossRef]

14. Bai, S.; Liu, X.; Zhu, K.; Wu, S.; Zhou, H. Metal-organic framework-based separator for lithium-sulfur batteries. Nano Energy 2016, 1, 16094. [CrossRef]

15. Song, R.; Fang, R.; Wen, L.; Shi, Y.; Wang, S.; Li, F. A trilayer separator with dual function for high performance lithium-sulfur batteries. J. Power Sources 2016, 301, 179-186. [CrossRef]

16. Guo, J.; Xu, Y.; Wang, C. Sulfur-impregnated disordered carbon nanotubes cathode for lithium-sulfur batteries. Nano Lett. 2011, 11, 4288-4294. [CrossRef] [PubMed]

17. Lee, J.S.; Manthiram, A. Hydroxylated N-doped carbon nanotube-sulfur composites as cathodes for high-performance lithium-sulfur batteries. J. Power Sources 2017, 343, 54-59. [CrossRef]

18. Chung, S.H.; Manthiram, A. High-Performance Li-S Batteries with an Ultra-lightweight MWCNT-Coated Separator. J. Phys. Chem. Lett. 2014, 5, 1978-1983. [CrossRef] [PubMed]

19. Li, Q.; Dong, L.; Fang, J.; Xiong, C. Property-Structure Relationship of Nanoscale Ionic Materials Based on Multiwalled Carbon Nanotubes. ACS Nano 2010, 4, 5797-5806. [CrossRef] [PubMed]

20. Bourlinos, A.B.; Herrera, R.; Chalkias, N.; Jiang, D.D.; Zhang, Q.; Archer, L.A.; Giannelis, E.P. Surface Functionalized Nanoparticles with Liquid-like Behavior. Adv. Mater. 2005, 17, 234-237. [CrossRef]

21. Li, J.; Huang, Y.; Zhang, S.; Jiang, W.; Wang, X.; Guo, Y.; Jia, D.; Wang, L. Decoration of Silica Nanoparticles on Polypropylene Separator for Lithium-Sulfur Batteries. ACS Appl. Mater. Interfaces 2017, 9, 7499-7504. [CrossRef] [PubMed]

22. Zu, C.; Su, Y.S.; Fu, Y.; Manthiram, A. Improved lithium-sulfur cells with a treated carbon paper interlayer. Phys. Chem. Chem. Phys. 2013, 15, 2291-2297. [CrossRef] [PubMed] 
23. Wu, F.; Chen, J.; Chen, R.; Wu, S.; Li, L.; Chen, S.; Zhao, T. Sulfur/Polythiophene with a Core/Shell Structure: Synthesis and Electrochemical Properties of the Cathode for Rechargeable Lithium Batteries. J. Phys. Chem. C 2011, 115, 6057-6063. [CrossRef]

24. Sun, J.; Huang, Y.; Wang, W.; Yu, Z.; Wang, A.; Yuan, K. Application of gelatin as a binder for the sulfur cathode in lithium-sulfur batteries. Electrochim. Acta 2008, 53, 7084-7088. [CrossRef]

25. Choi, J.-W.; Cheruvally, G.; Kim, D.-S.; Ahn, J.-H.; Kim, K.-W.; Ahn, H.-J. Rechargeable lithium/sulfur battery with liquid electrolytes containing toluene as additive. J. Power Sources 2008, 183, 441-445. [CrossRef]

26. Fu, Y.; Manthiram, A. Orthorhombic Bipyramidal Sulfur Coated with Polypyrrole Nanolayers as a Cathode Material for Lithium-Sulfur Batteries. J. Phys. Chem. C 2012, 116, 8910-8915. [CrossRef]

27. Zhou, G.; Pei, S.; Li, L.; Wang, D.W.; Wang, S.; Huang, K.; Yin, L.C.; Li, F.; Cheng, H.M. A graphene-pure-sulfur sandwich structure for ultrafast, long-life lithium-sulfur batteries. Adv. Mater. 2014, 26, 625-631. [CrossRef] [PubMed]

28. Wei, X.; Ma, J.; Li, B.; Zuo, Y.; Xia, D. Enhanced cycle performance of lithium-sulfur batteries using a separator modified with a PVDF-C layer. ACS Appl. Mater. Interfaces 2014, 6, 20276-20281. [CrossRef] [PubMed]

(C) 2017 by the authors. Licensee MDPI, Basel, Switzerland. This article is an open access article distributed under the terms and conditions of the Creative Commons Attribution (CC BY) license (http://creativecommons.org/licenses/by/4.0/). 